\title{
Pelvic organ prolapse in pregnancy
}

Volume 8 Issue 2 - 2017

\section{Introduction}

Pregnancy is a much-awaited joyous and momentous occasion. The delivery of a healthy infant to a likewise healthy mother brings about the satisfaction to both the family and the obstetrician. The care that is provided is tailored to fit the different needs that the pregnancy of every woman, to allow a safe delivery of an infant. The uncertainty of the course and outcome of each pregnancy leads to anticipation of every problem that may arise. This paper aims to address one such problem encountered by an increasing number of pregnant women, especially the multiparous parturient: Pelvic Organ Prolapse in pregnancy.

Pelvic organ prolapse has long been known as a disease of the multiparous elderly and postmenopausal. One recognized major risk factor also in the development of pelvic organ prolapse is vaginal birth, which confers a four- to eleven-fold increase in the development of prolapsed. ${ }^{1}$ The hypo-estrogenic state of menopause likewise has been identified as a major cause due to the subsequent weakening of the pelvic supports, with the median age of presentation is 61 years old, according to Luber et al. ${ }^{2}$ Other risk factors identified are body mass index, chronic constipation, and lifestyle. Inheritable risk of prolapse is also taken into consideration, as observed in nulliparous sisters of women with prolapsed. ${ }^{3}$ A study by Olsen et al in 1997 found that women with normal life expectancy have an $11 \%$ chance of undergoing at least one operation for pelvic organ prolapse in their lifetime. In the young adolescent, a congenital anomaly such as collagen defects may be the causative factor of the prolapse.

The rising number in literature of Pelvic Organ Prolapse in Pregnancy has been observed. However, little is known about the incidence of Pelvic Organ Prolapse during pregnancy, as there is a lack of documentation and standardization in the diagnosis. ${ }^{4}$ Historically, the treatment of prolapse, especially cervical prolapse, has evolved from interruption of pregnancy and setting fire to the cervix, to pessary use. ${ }^{5}$ The mounting awareness of the incidence of such disease entity in pregnancy has led to the need to document cases in order to have a deeper understanding, and in effect, possible prevention of future complications both during pregnancy and in advanced years after the pregnancy.

\section{Pelvic organ prolapse}

Pregnancy brings about anatomic, physiologic, and functional changes in the anticipation of the delivery of a normal infant. The spectrum of changes encompasses all systems in order to bring about a balanced and organized functional structure during pregnancy. One such system is the pelvic floor and its support. Little has been known about the changes that occur in the pelvic floor during pregnancy. ${ }^{4}$ Due to the impact of these changes either on the present pregnancy, or in future pregnancies, the need to document and to ascertain specific alterations from the normal becomes very apparent. ${ }^{6}$ Pelvic organ prolapse, urinary incontinence, and fecal incontinence are some of the few complications that have been noted as a result of childbirth, although poorly understood. The growing number has amplified the interest in determining their pathophysiology in relation to pregnancy. ${ }^{7}$

\author{
May Anne Tabaquero \\ St. Lukes Medical Center-Global City, Phillipines \\ Correspondence: May Anne Tabaquero, St. Lukes Medical \\ Center-Global City, Phillipines, Email may_md@yahoo.com \\ Received: April 25, 2017 | Published: October 30, 2017
}

Pelvic Organ Prolapse is defined as the descent of the pelvic organs into the vagina, often accompanied by urinary, bowel, sexual, or local pelvic symptoms. ${ }^{8}$ Prolapses are described according to the pelvic compartment involved, namely the anterior compartment (prolapse of the bladder and or the urethra), the middle compartment (uterine or vault descent, and enterocoele), and the posterior compartment (prolapse of the rectum). In a review of Stanton \& Thakar, ${ }^{8}$ cystourethrocoele was identified as the most common type of prolapse, followed by uterine type. Hence, urinary symptoms such as incontinence or voiding dysunctions are usually encountered in patients with prolapse.

DeLancey has also identified these defects according to the levels of support in 1992. He identified the three levels of pelvic support, the uterosacral-cardinal ligament complex (Level I), the paravaginal supports namely pubocervical and rectovaginal fascia (Level II), and perineal membrane and perineal body (Level III). The accompanying the symptomatology of the prolapse may be explained by the defects in the supports. The symptom common to all types of prolapse is usually an introital mass or vaginal bulge.

Pelvic Organ Prolapse is staged using the International Continence Society (ICS) Pelvic Organ Prolapse Quantification System (POP-Q) which was introduced in 1996. ${ }^{9}$ This staging presents a standard system of terminology approved by the International Continence Society, the American Urogynecologic Society, and the Society of Gynecologic Surgeons in describing the female pelvic organ prolapse and pelvic floor dysfunction. It is an objective site-specific system for describing, quantitating, and staging pelvic support in women is included. POP-Q introduces the 9-point grid, which identifies the different compartments of the pelvis (Figure 1).

One of the known risk factors in the development of Pelvic Organ Prolapse is pregnancy. There is no consensus that would directly correlate pregnancy and childbirth to the high rates of prolapse in parous women. However, many studies have postulated that pregnancy, labor, and vaginal delivery have a negative impact on the pelvic floor, 
especially the pudendal nerve, predisposing parous women to pelvic organ prolapse in the subsequent pregnancies. A review by Dietz and Wilson in 2005 addressed this issue by considering the following:

a. Adverse effects on nerve structure and function.

b. Adverse effects on pelvic floor muscle structure and functions.

c. Adverse effects on pelvic organ support

d. Epidemiological evidence linking childbirth to incontinence and prolapse.

e. It is also noteworthy to mention that changes in the pelvic support during pregnancy itself would predispose a parturient to prolapse during the pregnancy. ${ }^{10}$

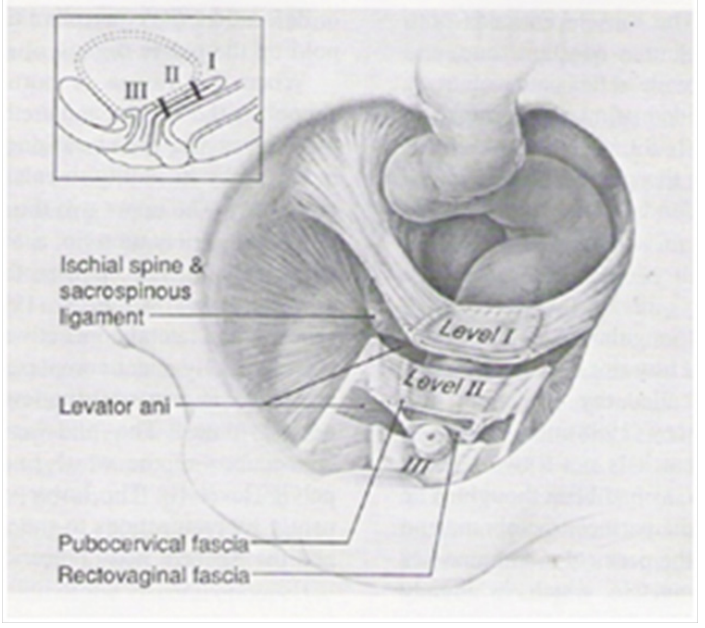

Figure I Levels of Pelvic Support. ${ }^{19}$

Changes in the pelvis and its supports have been to known to occur in order to accommodate the delivery of an infant vaginally and safely. The complex interplay of the alterations in the bony structure of the pelvis, ligamentous and connective tissue supports, musculature, and nerves bring about the systematic and organized process called delivery. ${ }^{5}$ The pelvis is a flexible basin in order to support the uterus with the growing fetus. The pelvic bones consisting of the ilium, ischium, pubic rami, sacrum, and coccyx, act synchronously to effect a foundation to which all the pelvic structures are ultimately anchored. In a non-pregnant woman, the pelvis is oriented such that the anterior superior iliac spine and pubic symphysis lie perpendicular to the pelvic floor, consequently, tilting the pelvic inlet anteriorly, with the genital hiatus parallel to the ground. This position directs the pressure of the intraabdominal and pelvic contents towards the pelvic bones, minimizing the pressure on the pelvic viscera and musculature. ${ }^{11}$ In pregnancy, these bony structures shift in order to redistribute the weight added by the gravid uterus, in order to maintain such orientation.

The spine, likewise, has been shown to assist the pelvis in the weight distribution during pregnancy. There is an observed exaggeration of the lumbar lordosis in pregnancy to maintain balance and equilibrium for the mother with her increasing abdominal girth. This physiologic lordosis that occurs allows the center of gravity of the mother to be maintained midline and deflects the weight of the pelvic viscera against the muscles of the anterior abdominal wall. This change leads to an increase in the anteroposterior diameter resulting in a vertically oriented pelvic inlet, decreasing incidence of pelvic organ prolapse. Thoracic kypohosis may likewise occur in pregnancy due to the increased weight of the breasts. Variations in the posture and subsequently changes in the bony pelvis are important factors that influence the maternal soft tissue damage and nerve injury during parturition. ${ }^{12}$ Some women are not able to achieve this curvature during pregnancy, and the loss of the lumbar lordosis produces a horizontally oriented pelvic inlet, wider transverse inlet, and narrowed anterior and posterior diameters, predisposing to prolapse.

The flaccidity of the abdominal musculoaponeurotic tissues increases with subsequent pregnancies. 5 Abdominal and pelvic relaxation coexist to allow an equal transmission of abdominal pressures due to pregnancy. With a further increase in the pelvic relaxation relative to the abdominal laxity, there is an increased pressure carried by the pelvic supports, predisposing to a prolapse during the present pregnancy, as well as future pregnancies. Soft tissue changes are due to deposition of hyaluronic acid that permits softening of the tissue structures due to its high water contents, and production of elastin that allows expansion of the tissues and remodeling of the supports. ${ }^{13,14}$ Parturition results in a distinct remodeling process especially of the vagina and cervix allowing their expansion. There is also a noted increase in the deposition of collagen, especially Types I and III that would contribute to this expansile property. The vagina adapts uniquely by an increase in production of mature elastic fibers, and recovery after delivery is likewise due to the regeneration of these fibers. ${ }^{14,15}$ The biochemical process that brings about the increase in the components such as hyaline permit the different soft tissues such as pelvic ligaments, endopelvic fascia, and soft tissue structures to stretch and to broaden. ${ }^{15}$ The vagina undergoes softening and thickening to be able to share and distribute the weight of the growing uterus evenly with the fascial and ligamentous tissues. By the processes of hypertrophy and hyperplasia, the uterine musculature adapts to the enlarging fetus. With advancing gestational age, the collagenolytic activity increases in order to effect delivery by cervical dilatation. In an article by Rinne and Kirkinen in 1998, they observed the importance of collagen during spontaneous labor, with a shortened course in women with low concentrations. ${ }^{2,16}$ In a patient with a decrease in collagen activity, pelvic relaxation as well as possible preterm birth may occur.

The net effect of these changes is pelvic relaxation, which may predispose the present pregnancy to a possible pelvic organ prolapse. Pelvic relaxation may be attributed to three factors namely the stretch (ligamentous and connective tissue supports, muscle), the impact on the neural pathways (pudendal nerve) and bony pelvis changes. ${ }^{10}$ The levator ani is a group of muscles (puborectalis, pubococcygeus, iliococcygeus) which acts to support the pelvic viscera such as the uterus. Studies have shown that the levator ani undergoes an increase in activity as the pregnancy progresses. In the early peripartal period, the levator ani activity is essentially the same prior to pregnancy up to the 8th week. However, the activity progressively increases significantly until delivery. The active basal tone keeps the urogenital hiatus closed and pelvic viscera over the levator plate. ${ }^{12}$ The contraction of the muscles decreases the tension placed on the connective tissues during increase in the intraabdominal pressure which is brought about by pregnancy. The stretch that occurs with the ligamentous and connective tissue supports such as the fascia and levator ani occur with difficult delivery, prolonged second stage of labor, and perineal trauma. It has been demonstrated in the study by Kearney et al in 2006 that the pubococcygeus is the part of the levator ani that undergoes the greatest degree of lengthening during vaginal delivery, due to muscle rupture and overstretching. ${ }^{17}$ 
The pudendal nerve undergoes compression during delivery leading to hypoxia, decreasing the transmission of impulses to the muscles it innervates, such as the levator ani, striated urethral sphincter, and external anal sphincter. The three stages of delivery may contribute to the susceptibility of pudendal nerve disruption and damage ${ }^{18}$ The birth-induced trauma to the levator ani secondary to pudendal nerve damage is considered the most modifiable risk factor in the development of pelvic organ prolapse, as interventions to prevent compression to the nerve structure during prolonged labor and descending head of the fetus may be instituted.

Stress incontinence may occur during pregnancy. Whether it is the pregnancy itself or the vaginal delivery which precede stress incontinence, the incidence of stress incontinence increases three fold with each subsequent delivery. ${ }^{19}$ Stress incontinence is primarily due to the decrease in the pelvic support provided by the Level III, or pubourethral ligaments, and the nerve disruption caused by the enlarging uterus on the pudendal nerve which would innervate the urethral sphincteric system.

Another striated muscle that is innervated by the pudendal nerve is the external anal sphincter. Aside from the damage brought about by the episiotomy, the pudendal nerve is again compressed against the descending head of the fetus. This may lead to fecal incontinece secondary to stretch and pudendal injury. In a study by Richter et al in 2006, they observed anal sphincter damage in $18 \%$ of vaginal deliveries on endoanal ultrasound, while $23-35 \%$ had occult damage, and was deemed symptomatic three months after delivery. Twenty nine to fifty three percent of the parturients with sphincter repair during delivery will report gas incontinence while 5-10\% developed stool incontinence. This emphasizes the need to evaluate the anal sphincters after delivery, especially those with prolonged and instrument-assissted vaginal births. ${ }^{20}$

Is the present pregnancy itself especially in the nulliparous predisposes to pelvic organ prolapse? Is the delivery process and the pelvic changes responsible? Will a pregnancy predispose the future ones to puerperal prolapses? What are the complications of a pregnancy that is accompanied by a pelvic organ prolapse? This is a case series about pelvic organ prolapse complicating a pregnancy. Pelvic organ prolapse was detected during the second trimester in all pregnancies. We follow the course of the prolapse as the pregnancies progressed, and how we addressed the complications brought about by the prolapse.

The first case is that of N.P., a 38 year old G2P1 (1001), who was first seen at $t$ nineteen weeks age of gestation. She was referred to the Urogynecology Service for an introital mass. Her first pregnancy was terminated via spontaneous vaginal delivery after eight hours lo labor, with an admitting cervical dilatation of six centimeters. She delivered to a live female, term, with a birthweight of seven pounds and five ounces in a government hospital. Five months after, she noted a $2 \times 2$ centimeter mass at the level of the introitus, especially during straining. Progressive increase in the size of the mass prompted consult in 1998, with a working impression of Uterine Prolapse. She was advised observation, with caution regarding weight bearing activities reiterated. No urinary complaints at this time.

On physical examination, it was noted that the cervix was protruding 2 centimeters from the hymen, with a cervical length of 5 centimeters (Figure 2). Impression at this time was Pelvic Organ Prolapse III, the leading point being the cervix. Review of history of the present pregnancy revealed two episodes of urinary retention during her first month, with catheterization done. On her twentieth week of pregnancy, she was fitted with a pessary, and advised to continue use until her $32^{\text {nd }}$ to $34^{\text {th }}$ week, after which the pessary was to be removed to allow spontaneous onset of labor. She was taught pessary insertion and care. Monthly follow-up was done, together with the Perinatology Service. Estrogen cream was applied, and oral tocolytics given on a PRN basis.

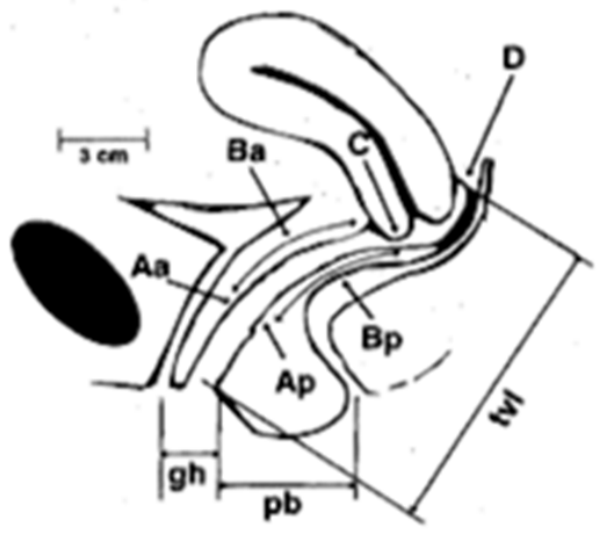

Figure 2 POP-Q Staging.

On her $34^{\text {th }}$ week of pregnancy, a re-evaluation of the prolapse was done, which revealed a Stage II prolapse, the cervix noted to be one centimeter above the hymen (Figure 3). She eventually delivered by classical cesarean section secondary to malpresentation (transverse lie) with bilateral tubal ligation to a live term female with a weight of 2800 grams. Upon discharge, the cervix was noted to be still one centimeter above the hymen. The patient has been advised re-evaluation of prolapse stage six weeks after delivery, and consideration of a definitive procedure for treatment of the prolapse was presented (Table $1 \& 2$ ).

Table I ICS Scoring (At I4 weeks)

\begin{tabular}{lll}
\hline Aa -2 & Ba -2 & C +2 \\
\hline GH 6 & PB 2 & TVL 8 \\
Ap -3 & BP -3 & D -3 \\
\hline
\end{tabular}

Table 2 ICS Scoring (Post-partum)

\begin{tabular}{lll}
\hline Aa -2 & Ba -2 & C -2 \\
\hline GH 5 & PB 2 & TVL 7 \\
Ap -2 & Bp -2 & D -4 \\
\hline
\end{tabular}

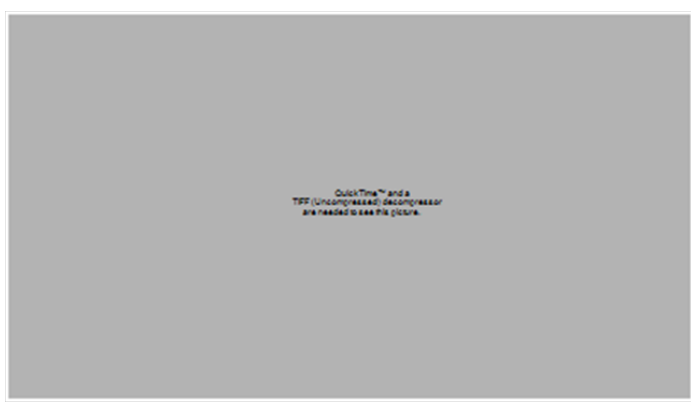

Figure 3 Pessary Insertion at 20 weeks.

The second case is that of M.S., 38 year old G3P2 (2002), who was first seen at sixteen weeks age of gestation. She presented at the OB admitting section for an introital mass which was noted to be the cervix descended up to the level of the hymen. Internal examination revealed closed cervix. The impression at that time was Pelvic Organ Prolapse 
II. She was discharged and referred to the Urogynecology Service on an outpatient basis. At the Outpatient Department, the patient was re-evaluated, and Stage II Prolapse was maintained. The cervix was noted to be effaced at this time, with complaints of occasional hypogastric pain and uterine contractions. The patient was referred back to the Perinatology Service for serial cervical evaluation and monitoring. Effacement and funneling were not seen on ultrasound, but more frequent prenatal follow up was advised.

The patient's two previous pregnancies (G1 1997, G2 2000) were terminated vaginally at home assisted by a traditional birth attendant. Her first baby weighed seven pounds, and her length of labor was approximately eight hours. Her second baby weighed seven pounds and three ounces, and length of labor was approximately seven hours. She noted presence of the mass only during her third pregnancy, although she claimed to have had pelvic heaviness prior to her second pregnancy (Table 3). On her $28^{\text {th }}$ week of gestation, a right ovarian mass was seen on routine pelvic ultrasound. She was admitted with an impression of Ovarian New Growth in pregnancy. She underwent Right Salpingo-oophorectomy, and histopathology revealed Serous Cystadenoma. Referral was made at this time, and re-evaluation of prolapse was done. The cervix, which was previously the leading point, was noted to be 2 centimeters above the hymen, and the prolapse was downstaged to Stage I. She was discharged with oral tocolytics, and placed on bed rest.

Table 3 ICS Scoring (At 20 weeks age of gestation)

\begin{tabular}{lll}
\hline Aa 0 & Ba 0 & C -2 \\
\hline GH 5 & PB 2 & TVL 9 \\
Ap - I & Bp - I & D -4 \\
\hline
\end{tabular}

The rest of her pregnancy was unremarkable, and she delivered vaginally on her $38^{\text {th }}$ week of gestaion, with bilateral tubal ligation, live term female, with a birthweight of 3800 grams. Stage at this time remained at Stage I (Figure 4). Re-evaluation of the prolapse will be done six weeks after delivery (Table 4).

Table 4 ICS Scoring (Postpartum)

\begin{tabular}{lll}
\hline Aa -2 & Ba -2 & C -4 \\
\hline GH 5 & PB 2 & TVL 9 \\
Ap - I & Bp - I & D -6 \\
\hline
\end{tabular}

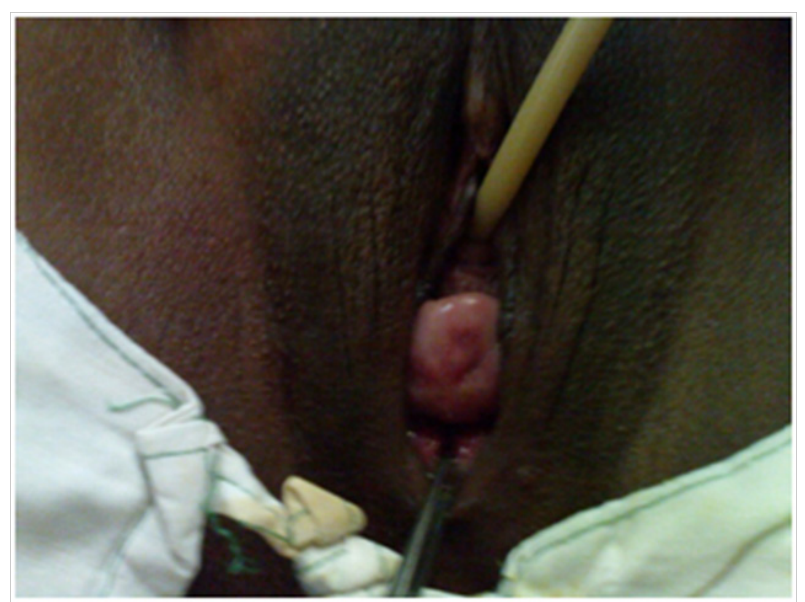

Figure 4 Prolapse Prior to Abdominal Sacrohysteropexy, Burch Colposuspension, Posterior Repair.

The third case is that of M.B., a 26 year old G3P2 (2002) who was first seen at fourteen weeks age of gestation (Figure 5). She was seen at the OB Admitting Section with a complaint of an introital mass. Impression at this time was Pelvic Organ Prolapse. Referral to Urogynecology was done during this consult. Staging of her prolapse at this time revealed a Stage III Pelvic Organ Prolapse, with cervix as the leading point. Incidentally, urinary tract infection was diagnosed, and she was given antibiotic coverage. The patient was lost to follow up with the Urogynecology Service. She claimed to have an uneventful prenatal course, with no preterm labor nor urinary symptoms. She eventually delivered vaginally to a female, term, with a birth weight of seven pounds (Table 5).

Table 5 ICS Scoring (At I4 weeks Age of Gestation)

\begin{tabular}{lll}
\hline $\mathbf{A a} \mathbf{0}$ & Ba 0 & C +2 \\
\hline GH 5 & PB 3 & TVL 8 \\
Ap -2 & Bp -2 & D -3
\end{tabular}

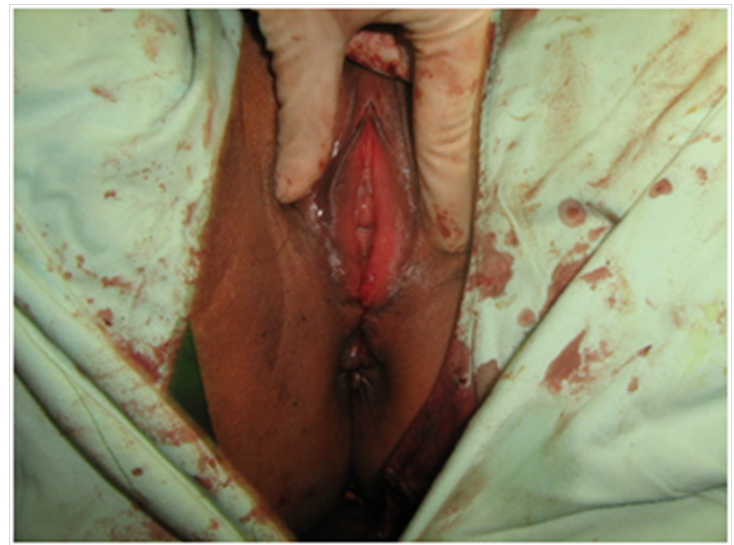

Figure 5 s/p Abdominal Sacrohysteropexy with Burch Colposuspension and Posterior Repair.

On review of her history, it was noted that the length of labor of her first pregnancy was approximately twelve hours, with rupture of bag of waters as the reason of admission. Cervical dilatation at that time was 4 centimeters. Slow progress of labor was assumed, and length of second stage of labor was approximately three hours. Birth weight of the first child was six pounds and four ounces. Second pregnancy was uneventful, although patient claimed that there was already note of pelvic organ descent in the form of cervical descent.

Seven months after her delivery, she presented at the OPD with a complaint of introital mass. Restaging of the prolapse at this time revealed a Stage II prolapse, with the cervix protruding one centimeter beyond the hymen (Figure 6). She was advised Abdominal Sacrohysteropexy with Burch Colposuspension and Posterior Repair. She is presently restaged at Stage $\mathrm{O}$ after the procedure (Table $6 \&$ 7).

Table 6 ICS Scoring (Prior to Abdominal Sacrohysteropexy)

\begin{tabular}{lll}
\hline Aa 0 & Ba 0 & C + I \\
\hline GH 4 & PB 3 & TVL 6 \\
Ap -3 & Bp -2 & D -2 \\
\hline
\end{tabular}

Table 7 ICS Scoring (s/pAbdominal Sacrohysteropexy, Burch Colposuspension, Posterior Repair)

\begin{tabular}{lll}
\hline Aa -3 & Ba -3 & C -4 \\
\hline GH 4 & PB 3 & TVL 6 \\
Ap -3 & BP -3 & D -6 \\
\hline
\end{tabular}

All three cases presented with Pelvic Organ Prolapse in pregnancy, with the cervix as the leading point of the descent. All three patients were multiparous, the two having history of protracted labor, one 
having a history of large birthweights. Connective tissue diseases were not present in all three patients. The three patients noted the presence of the introital mass or pelvic heaviness after their first pregnancy. Stress incontinence and fecal incontinence were not present.

The low incidence of Pelvic Organ Prolapse complicating a pregnancy is probably due to lack of proper documentation and reporting. However, due to the increasing awareness and better understanding of the pathophysiology of Pelvic Organ Prolapse, coinciding with the use of a uniform scoring system, the incidence of this disease entity is slowly rising. Pregnancy has long been known as a risk factor in the development of the Pelvic Organ Prolapse, although its detection is done during the postmenopausal years, where the other factors that affect pelvic support come into play. Pelvic organ prolapse in pregnancy is considered high risk due to the risk of early and late prenatal losses or premature labor. Other observed complications may include urinary tract infection, urinary retention, maternal sepsis are some of the more severe events that can be correlated with pelvic organ prolapse. Cervical prolapse, or pelvic organ prolapse with the cervix as the identified most descended portion, is the type that occurred in the patients described previously. In a case report by Sawyer, et al in 1999, less than 300 cases have been reported. The complications observed in these cases were patient discomfort due to cervical dessication and ulcerations, which may ultimately lead to maternal sepsis. Likewise, the protrusion of the cervix may give way to cervical dilatation and subsequent effacement. Referral to Perinatology Service should be done for serial monitoring of cervical effacement and dilatation, and infection of the prolapsing compartment.

The most frequent identified complication of pelvic organ prolapse in pregnancy is preterm labor. Increased turnover of elastic fibers with massive remodeling occur during pregnancy and birth. However, due to the presence of increased expression of elastin relative to the turnover, there is maintenance of elastic fiber homeostasis. In some pregnancies, there is a deficiency in the LOXL1 leading to production of elastase. Manifestations include cervical insufficiency leading to early pregnancy losses and preterm labor due to an inherent weakness of the cervix. The three patients were able to bring their pregnancies to term without complaints of preterm labor. ${ }^{15}$

Another manifestation due to the imbalance of elastic fiber homeostasis is increased incidence of stress incontinence. Parallel to cervical insufficiency, the support structures of the urethral sphincteric mechanism is weakened, leading to the inability of the sphincter to maintain a water tight seal in preventing urinary leakage. Stress incontinence may also be secondary to the increase in the descent of Point Aa in the ICS scoring which may lead to greater urethral mobility, hence increase in the frequency of stress incontinence episodes as the gestation advances, peaking in the third trimester. ${ }^{19}$ The patients did not complain of stress incontinence. The most descended compartment was the middle compartment, and evaluation of the prolapses prior to delivery showed a non-prominent anterior vaginal wall.

The use of pessary is one mode of treatment that can be used in pregnancy complicated by a prolapse, especially that of a cervical prolapse. Historically, cervical prolapse was treated with interruption of pregnancy due to the high incidence of early and midpregnancy losses. The development of the Smith-Hodgkin's pessary allowed the management of the cervical prolapse by restoring the normal position of the cervix above the hymen by supporting it between the symphysis pubis and coccyx, acting as the pericervical support. ${ }^{5}$ Other type of pessaries may be used in conjunction with symptoms of stress incontinence, by means of obstruction at the sphincteric level, acting as periuretheral supports. These types of pessaries allow efficient closure of the sphincter by virtue of obstruction during increases in intraabdominal pressure.

In a survey done by Cundiff et al. ${ }^{21}$ regarding the use of the pessary by the members of the American Urogynecological Society, $77 \%$ used pessaries as first line of therapy for prolapse (48\% response rate), while $12 \%$ reserved the use of pessaries for patients who were not surgical candidates which may include puerperal prolapsed..$^{21}$ The use of pessary in pregnancy is done likewise to prevent the shortening of the cervix leading to spontaneous preterm birth secondary to cervical incompetence or puerperal prolapse. It supports the cervix in pregnant patients with prolapse by directing the cervix in its normal anatomic orientation, or posteriorly changing the inclination of the canal. The pessary then directs the weight of the gravid uterus towards the anterior lower segment preventing cervical dilatation and premature rupture of membranes secondary to the prolapsed. ${ }^{22}$

Disadvantages of pessary use would be undue manipulation of the cervix that may lead to uterine stimulation, subsequently preterm labor. Oral tocolytics may be started with patients who use pessaries to avoid this event. Close monitoring of the patient during the period of pessary use is warranted. Conservative management such as close observation and adequate bed rest, and expectant management, is also a modality that can be initiated to prevent further complications due to the descent of the pelvic organs. This was instituted in the second patient where in close observation and initiation of treatment when necessary proved beneficial. In her case, it was noted that the descent of the cervix did not progress to a higher stage. Serial cervical evaluation was done because of the initial examination. The cervix was observed to be two centimeters above the hymen, probably due to the uterus becoming an abdominal organ during the second trimester, and the shortening of the cervix due to the enlarging uterus. Manipulation of the prolapse which would lead to uterine hyperstimulation should be avoided. Administration of corticosteroids for fetal lung maturation may be done, as anticipation to a preterm delivery.

Definitive management after delivery may be based on the woman's decision whether to maintain her reproductive function. In the case of the patient who underwent abdominal sacrohysteropexy with Burch Colposuspension and Posterior Repair, she opted to maintain her reproductive career, taking into consideration her age and ovarian status. The patient is 26 years of age, and removal of her uterus even with a completed family would imply early menopause. The severity of her prolapse at such an early age compared to the mean would imply either a congenital abnormality such as collagen defects, or severe trauma of pelvic support structures during her first and second pregnancy. The history or prolonged labor during the first and second deliveries may point out to levator ani damage, either due to stretch or pudendal nerve injury. Because of the acquired weakness and the inherent changes of the pelvic support in pregnancy, the further descent of the prolapse relative to the one reported during the second pregnancy.

Abdominal sacrohysteropexy is a compensatory procedure where in the uterus is preserved while addressing the problem of a uterovaginal prolapse by the use of mesh, which in this case, was synthetic. According to a study by Demirci et al., ${ }^{23}$ this type of prolapse surgery is considered effective and safe in the treatment of uterovaginal prolapse for women who is still desirous of pregnancy and of retaining their uteri. It provides a durable anatomic restoration by maintaining the vaginal length and the normal axis of the vagina, and normal sexual function with minimal complications. 
A study in 2001 conducted by Barranger, Fritel, and Pigne wherein thirty women of childbearing age underwent sacrohysteropexy with Burch colposuspension and posterior repair had no recurrence of uterovaginal prolapse after long term follow-up of 94.6 months without a time-dependent decrease in efficiency. ${ }^{24}$ The accompanying site-specific management of the anterior and posterior prolapse include Burch Colposuspension and Posterior Repair with Levator Myorrhaphy. The Burch Colposuspension was primarily done to address the anterior defect by preferentially elevating the anterior vaginal wall and bladder neck, and anchoring them to the Cooper's Ligament. The posterior repair with levator myorrhapy addressed the posterior compartment by repairing and strengthening the rectovaginal fascia.

Proper prolapse care was likewise advised in all three patients. Because of the exposure of the cervix as it protrudes out of the hymen, desiccation and ulceration may occur as a consequence. ${ }^{5}$ The use of a topical estrogen would allow the cervix to be adequately hydrated by promoting good blood supply, hence preventing ulcerations if present. The severity of the ulceration may subsequently lead to maternal sepsis, translating to neonatal sepsis upon delivery with the active infection present.

Aside from the probable complications of the prolapse during a pregnancy, one should also consider the after effects of the pregnancy leading to the prolapse. Understanding of the role of vaginal birth and pelvic organ prolapse is limited due to the long interval between the delivery and increased risk of pelvic organ prolapse. As seen in levator ani muscle injuries, the lack of a standardized measure has led to the under-reporting of pelvic organ prolapse, and fecal and urinary incontinence. However, due to the introduction of the standardized clinical measurement of POP-Q System, evaluation of women soon after may be done, leading to an increase in the reporting of such effect.

Evaluation of the prolapse every trimester may be warranted for patients with pelvic organ prolapse, in order to assess any increase or decrease in the POP-Q staging, suggesting alterations in pelvic support during the prenatal period and prior to delivery. In order to focus specifically on the influence of pregnancy may be addressed by evaluation in each and every trimester. ${ }^{7}$ Elucidating the changes which occur in puerperal prolapse and identifying the obstetric variables may help contribute to the development of protocols and interventions such as pelvic floor exercises and elective cesarean section to prevent prolapses in the future.

\section{Conclusion}

Treatment of pelvic organ prolapse in pregnancy should be individualized to fit the needs and lifestyle of the patient. There is not one algorithm that should be followed as every pregnancy would differ from the next. We should take into consideration the present status of the prolapse and plan for a definitive management if warranted after delivery.

Pelvic Organ Prolapse is not limited to the elderly and postmenopausal women. Active women who are in the prime of their lives may be affected. Risk factors include multiple vaginal deliveries leading to levator ani muscle injury and pelvic changes itself caused by pregnancy are identified in these relatively young women. If these deleterious effects are maintained even after delivery, the consequence of the pregnancy becomes very apparent in succeeding pregnancies, and unfortunately, their lifestyle. The need to evaluate all parturients during prenatal examination for the presence of a prolapse cannot be overemphasized. The impact of the prolapse and its implications to a pregnancy should be studied further for formulation of better treatment modalities, and in the future, prevention.

\section{Acknowledgments}

None.

\section{Conflicts of interest}

None.

\section{References}

1. Rinne, Kirsi M, Pertti P Kirkinen. What Disposes Young Women to Genital Prolapse? Original Article. Europ J Obstet Gynecol and Repro Bio. 1999;84:23-25.

2. Luber KM, Boero S, Choe JY. The Demographics of Pelvic Floor Disorders: Current Observations and Future Projections. Am J Obstet Gynecol. 2001;184(7):1496-1501.

3. Buchsbaum GM, Duecy EE, Kerr LA, et al. Pelvic Organ Prolapse in Nulliparous Women and Their Parous Sisters. Obstet Gynecol. 2006;108(6):1288-93.

4. Olsen AL, Smith VJ, Bergstrom JO, et al. Epidemiology of Surgically Managed Pelvic Organ Prolapse and Urinary Incontinence. Obstet Gynecol. 1997;89(4):501-506.

5. Sawyer Devin, Keith Frey. Cervical Prolapse During Pregnancy. $J$ Am Board of Family Pract. 2000;13(3):216-218.

6. Sze EH, Sherard GB, Dolezal JM Pregnancy, Labor, Delivery, and Pelvic Organ Prolapse. Obstet Gyneco. 2002;1 100(5 Pt 1): 981-986.

7. O Boyle AL, O Boyle JD, Ricks RE, Patience TH, Calhoun B, et al. The Natural History of Pelvic Organ Support in Pregnancy. Int Urogynecol J. 2003; 14(1): 46-49.

8. Thakar R, Stanton S Management of Genital Prolapse. BMJ. 2002; 324(7348):1258-1262

9. Bump RC, Mattiasson A, Bø K, Brubaker LP, De Lancey JO, et al. The standardization of terminology of female pelvic organ prolapse and pelvic floor dysfunction. Am J Obstet Gyneco. 1996;1 175(1): 10-17.

10. Dietz HP, Wilson PD Childbirth and Pelvic Floor Trauma. Best Pract Res Clin Obstet Gynaecol. 2005; 19(6): 913-924.

11. Barber Contemporary Views on Female Pelvic Anatomy. Cleve Clin J Med 72 (Suppl 4): S3-S11. 2005

12. Nguyen JK, Lind LR, Choe JY, et al. Lumbosacral Spine and Pelvic Inlet Changes Associated with Pelvic Organ Prolapse. Obstet Gynecol. 2000;95(3):332-336.

13. Drewes PG, Yanagisawa H, Starcher B, et al. Pelvic Organ Prolapse in Fibulin-5 Knockout Mice: Pregnancy Induced Changes in Elastic Fiber Homeostais in Mouse Vagina. The Am J Pathol. 2007;170(2):578-589.

14. Liu X, Zhao Y, Pawlyk B, et al. Failure of Elastic Fiber Homeostasis Leads to Pelvic Floor Disorders. Am J Pathol. 2006;168(2):519-528.

15. Samuel CS, Coghlan JP, Bateman JF. Effects of Relaxin, Pregnancy, and Parturition on Collagen Metabolism in the Rat Pubic Symphysis. $J$ Endocrinol. 1998;159(1):177-125.

16. Berlowitz, Lori. A 46-Year-Old Woman with Pelvic Floor Relaxation After a Second Vaginal Delivery. N Engl J Med. 2004350:2699-2706.

17. Kearney R, Miller JM, Ashton-Miller JA, et al. Obstetric Factors Associated with Levator Ani Muscle Injury After Vaginal Birth. Obstet Gynecol. 2006;107(1):144-149. 
18. Guaderrama NM, Liu J, Nager CW, et al. Evidence for the Innervation of Pelvic Floor Muscles by the Pudendal Nerve. Obstet Gynecol. 2005;106(4):774-781.

19. DeLancey JO, Morgan DM, Fenner DE, et al. Comparison of Levator Ani Muscle Defects and Function in Women With and Without Pelvic Organ Prolapse. Obstet Gynecol. 2007;109(2 Pt 1):295-302.

20. Richter HE, Fielding JR, Bradley CS, et al. Endoanal Ultrasound Findings and Fecal Incontinence symptoms in Women With and Without Recognized Anal Sphincter Tears. Obstet Gynecol. 2006;108(6):139499.

21. Cundiff GW, Weidner AC, Visco AG, et al. A Survey of Pessary Use by Members of the American Urogynecologic Society. Obstet Gynecol. 2000;95:931-935.
22. Arabin B, Halbesma JR, Vork F, et al. Is Treatment with Vaginal Pessaries an Option in Patients With a Sonographically Detected Short Cervix? J Perinat. 2003;31(2):122-133.

23. Demirci F, Ozdemir I, Somunkiran A, et al. Abdominal Sacrohysteropexy in Young Women with Uterovaginal Prolapse: Results of 20 Cases. $J$ Reprod Med. 2006;51(7):539-543.

24. Barranger E, Fritel X, Pigne A. Abdominal Sacrohysteropexy in Young Women with Uterovaginal Prolapse: Long Term Follow-up. J Obstet Gynecol. 2003;189(5):1245-1250. 